\title{
Ring Derivations on Semi-Simple Commutative Banach Algebras
}

\author{
Osamu HATORI and Junzo WADA
}

Tokyo Medical College and Waseda University

\section{Introduction.}

Let $A$ be a commutative Banach algebra. An (resp. linear) operator $D$ on $A$ is called a ring (resp. linear) derivation on $A$ if equations $D(f+g)=D(f)+D(g)$ and $D(f g)=f D(g)+D(f) g$ are satisfied for every $f$ and $g$ in $A$. The image of linear derivation was studied by Singer and Wermer [5] under the hypothesis of continuity of the operator, and Thomas [6] has proved that every linear derivation on a commutative Banach algebra maps into the radical of the algebra. On the other hand there are ring derivations which do not map into the radical (cf. [1]). In this paper we characterize ring derivations on semi-simple commutative Banach algebras. A function algebra is semi-simple and so the results generalize our previous results in [3]. As a consequence of the results it is shown that only the zero operator is a ring derivation on a semi-simple commutative Banach algebra with the carrier space without an isolated point, which is a generalization of a theorem of Nandakumar [4].

\section{Lemmata.}

LEMMA 1. Let $A$ be a commutative Banach algebra with the carrier space $M_{A}$. Suppose that $D$ is a ring derivation on $A$. Then $(D(\alpha f))^{\wedge}=\alpha(D(f))^{\wedge}$ for every $f$ in $A$ and for every rational number $\alpha$ in the complex number field $C$, where ${ }^{*}$ denotes the Gel'fand representation.

Proof. If $\alpha$ is a rational real number, then $D(\alpha f)=\alpha D(f)$ by standard argument. So we only show that $(D(i f))^{\wedge}=i(D(f))^{\wedge}$, where $i$ is the imaginary unit. For every $f$ in $A$,

$$
2 f D(f)=D\left(f^{2}\right)=-D\left((i f)^{2}\right)=-2 i f D(i f),
$$

so we have $(D(f))^{\wedge}(x)=-i(D(i f))^{\wedge}(x)$ for every $x$ in $M_{A}$ with $\hat{f}(x) \neq 0$. When $\hat{f}(x)=0$, choose $g$ in $A$ with $\hat{g}(x) \neq 0$. In the same way we have $(D(g))^{\wedge}(x)=-i(D(i g))^{\wedge}(x)$ and $(D(f+g))^{\wedge}(x)=-i(D(i(f+g)))^{\wedge}(x)$ since $(f+g)^{\wedge}(x)=\hat{f}(x)+\hat{g}(x) \neq 0$, so

$$
(D(f))^{\wedge}(x)+(D(g))^{\wedge}(x)=-i(D(i f))^{\wedge}(x)-i(D(i g))^{\wedge}(x) .
$$


We conclude that $(D(f))^{\wedge}(x)=-i(D(i f))^{\wedge}(x)$ even if $\hat{f}(x)=0$. It follows that $i(D(f))^{\wedge}$ $=(D(\text { if }))^{\wedge}$ on $M_{A}$.

REMARK. If $A$ contains the unit, then $D(\alpha f)=\alpha D(f)$ for every $f$ in $A$ and rational complex number $\alpha$. But it is not the case when $A$ is not unital. Let $C$ be the complex number field. Then $C$ is a radical Banach algebra under the usual scalar multiplication and the usual summation and the multiplication $\times$ defined by $a \times b=0$ with the norm $\|\cdot\|=|\cdot|$. Define $D$ by $D(a)=\bar{a}$, then $D$ is a ring derivation and $D(i a) \neq i D(a)$ if $a \neq 0$.

LEMMA 2. Let $A$ be a commutative Banach algebra with the carrier space $M_{A}$. Suppose that $x$ and $y$ are different points in $M_{A}$. Then there is $f$ in $A$ with $f(x)=0$ and $\hat{f}(y)=1$.

\section{Proof is trivial.}

LEMMA 3. Let $A$ be a commutative Banach algebra with the carrier space $M_{A}$. Let $\left\{x_{n}\right\}$ be a sequence of distinct points in $M_{A}$. Suppose that $D$ is a ring derivation on $A$. There is $f_{1}$ in $A$ which satisfy that $\left\|f_{1}\right\|_{A} \leqq 1 / 2,\left\|D\left(f_{1}\right)\right\|_{A} \leqq 1 / 2$ and $\hat{f}_{1}\left(x_{i}\right) \neq 0$ for every positive integer $i$. For every positive integer $n$ greater than 1 there is $f_{n}$ in $A$ which satisfies that $\left\|f_{n}\right\|_{A} \leqq 1 / 2,\left\|D\left(f_{n}\right)\right\|_{A} \leqq 1 / 2, \hat{f}_{n}\left(x_{i}\right)=0$ for $1 \leqq i<n$ and $\hat{f}_{n}\left(x_{i}\right) \neq 0$ for $n \leqq i$.

We can prove Lemma 3 by the same way as in the proof of Lemma 2 in [2].

LEMMA 4. Let $A$ be a commutative Banach algebra with the carrier space $M_{A}$. If the (not necessarily linear) functional $\phi_{x}(f)=(D(f))^{\wedge}(x)$ defined on $A$ is not continuous, then for every pair of positive numbers $\varepsilon$ and $K$ there exists $f$ in $A$ such that $\|f\|_{A}<\varepsilon$ and $\left|(D(f))^{\wedge}(x)\right|>K$.

Proof. Suppose that there are positive number $\varepsilon_{0}$ and $K_{0}$ which satisfy that for every $f$ in $A$ with $\|f\|_{A}<\varepsilon_{0}$ we have $\left|(D(f))^{\wedge}(x)\right| \leqq K_{0}$. We will show that $\phi_{x}$ is continuous. Let $\delta$ be a positive number. Put $\varepsilon=\delta^{\prime} \varepsilon_{0} / K^{\prime}$, where $\delta^{\prime}$ and $K^{\prime}$ are rational positive numbers such that $\delta^{\prime}<\delta$ and $K_{0}<K^{\prime}$. If $\|f\|_{A}<\varepsilon$, then $\left\|\left(K^{\prime} / \delta^{\prime}\right) f\right\|_{A}<\varepsilon_{0}$ so $\left|\left(D\left(\left(K^{\prime} / \delta^{\prime}\right) f\right)\right)^{\wedge}(x)\right| \leqq$ $K_{0}$. Since $D$ is linear over rational real number field, which is proven by the standard argument, we have $D\left(\left(K^{\prime} / \delta^{\prime}\right) f\right)=\left(K^{\prime} / \delta^{\prime}\right) D(f)$ and so $\left|(D(f))^{\wedge}(x)\right|<\delta$, which means that $\phi_{x}$ is continuous at 0 . Thus we see that $\phi_{x}$ is continuous since $D(f-g)=D(f)-D(g)$ for every $f$ and $g$ in $A$.

The following lemma is a version of Theorem 1 in [2] in the case of ring derivations on Banach algebras.

LEMMA 5. Let $A$ be a commutative Banach algebra with the carrier space $M_{A}$. Let $D$ be a ring derivation on $A$. Then the functional $\phi_{x}(f)=(D(f))^{\wedge}(x)$ on $A$ is continuous for every $x$ in $M_{A}$ but a finite exceptions.

Proof. Suppose that there are infinite number of points $x$ in $M_{A}$ at which $\phi_{x}$ is not continuous. Choose a sequence $\left\{x_{n}\right\}$ of distinct points at which $\phi_{x}$ is discontinuous. 
For the sequence $\left\{x_{n}\right\}$, choose a sequence $\left\{f_{n}\right\}$ in $A$ which satisfies the conditions in Lemma 3. Define inductively a sequence $\left\{F_{n}\right\}$ in $A$ as follows. Put $F_{1}=0$. If $F_{1}, \cdots, F_{i-1}$ is defined, then put $F_{i}$ in $A$ satisfying the conditions:

1) $\left\|F_{i}\right\|_{A}<1$

2) $\left|\left(D\left(F_{i}\right)\right)^{\wedge}\left(x_{i}\right)\right|>\left(i+\left|\left(D\left(\sum_{j=1}^{i-1}\left(\prod_{i=1}^{j} f_{\imath}^{2}\right) F_{j}\right)\right)^{\wedge}\left(x_{i}\right)\right|\right) /\left|\prod_{j=1}^{i}\left(\hat{f}_{j}^{2}\left(x_{i}\right)\right)\right|$.

We see that $\left\|D\left(\prod_{j=1}^{i} f_{j}^{2}\right)\right\|_{A} \leqq 1 / 2$ for every $i$ by induction on $i$. If $i=1$, then

$$
\begin{aligned}
\left\|D\left(f_{1}^{2}\right)\right\|_{A} & =\left\|2 f_{1} D\left(f_{1}\right)\right\|_{A} \\
& \leqq 2\left\|f_{1}\right\|_{A}\left\|D\left(f_{1}\right)\right\|_{A} \\
& \leqq 1 / 2 .
\end{aligned}
$$

We will show that $\left\|D\left(\prod_{j=1}^{i+1} f_{j}^{2}\right)\right\|_{A} \leqq 1 / 2$ under the hypothesis that $\left\|D\left(\prod_{j=1}^{i} f_{j}^{2}\right)\right\|_{A} \leqq 1 / 2$.

$$
\begin{aligned}
\left\|D\left(\prod_{j=1}^{i+1} f_{j}^{2}\right)\right\|_{A} & =\left\|f_{i+1}^{2} D\left(\prod_{j=1}^{i} f_{j}^{2}\right)+\left(\prod_{j=1}^{i} f_{j}^{2}\right) 2 f_{i+1} D\left(f_{i+1}\right)\right\|_{A} \\
& \leqq\left\|f_{i+1}\right\|_{A}^{2}\left\|D\left(\prod_{j=1}^{i} f_{j}^{2}\right)\right\|_{A}+2\left(\prod_{j=1}^{i}\left\|f_{j}\right\|_{A}{ }^{2}\right)\left\|f_{i+1}\right\|_{A}\left\|D\left(f_{i+1}\right)\right\|_{A} \\
& \leqq 1 / 2 .
\end{aligned}
$$

Put

$$
G=\sum_{i=1}^{\infty}\left(\prod_{j=1}^{i} f_{j}^{2}\right) F_{i}
$$

and

$$
G_{p}=\sum_{i=p+1}^{\infty}\left(\prod_{j=1, j \neq p+1}^{i} f_{j}^{2}\right) F_{i} .
$$

Then $G$ and $G_{p}$ converge in $A$ since $\left\|f_{j}\right\|_{A} \leqq 1 / 2$ and $\left\|F_{i}\right\|_{A}<1$. We see that

$$
G=\sum_{i=1}^{p}\left(\prod_{j=1}^{i} f_{j}^{2}\right) F_{i}+f_{p+1}^{2} G_{p}
$$

We will show that

$$
\left|(D(G))^{\wedge}\left(x_{p}\right)\right| \geqq p-1
$$

for every positive integer $p$. This is trivial for $p=1$, so we will prove it for $p \geqq 2$. Since $\hat{f}_{p+1}\left(x_{p}\right)=0$ for every $p$ we have

$$
\begin{aligned}
\left(D\left(f_{p+1}^{2} G_{p}\right)\right)^{\wedge}\left(x_{p}\right) & =\hat{f}_{p+1}\left(x_{p}\right)\left(D\left(f_{p+1} G_{p}\right)\right)^{\wedge}\left(x_{p}\right)+\hat{f}_{p+1}\left(x_{p}\right) \hat{G}_{p}\left(x_{p}\right)\left(D\left(f_{p+1}\right)\right)^{\wedge}\left(x_{p}\right) \\
& =0
\end{aligned}
$$

Thus 


$$
\begin{aligned}
\left|(D(G))^{\wedge}\left(x_{p}\right)\right|= & \left|\left(D\left(\sum_{i=1}^{p}\left(\prod_{j=1}^{i} f_{j}^{2}\right) F_{i}\right)\right) \hat{)}\left(x_{p}\right)\right| \\
\geqq & \left|\left(D\left(\left(\prod_{j=1}^{p} f_{j}^{2}\right) F_{p}\right)\right) \hat{n}\left(x_{p}\right)\right|-\left|\left(D\left(\sum_{i=1}^{p-1}\left(\prod_{j=1}^{i} f_{j}^{2}\right) F_{i}\right)\right) \hat{(}\left(x_{p}\right)\right| \\
\geqq & \left|\left(\prod_{j=1}^{p} \hat{f}_{j}^{2}\left(x_{p}\right)\right)\left(D\left(F_{p}\right)\right)^{\wedge}\left(x_{p}\right)\right|-\left|\hat{F}_{p}\left(x_{p}\right)\left(D\left(\prod_{j=1}^{p} f_{j}^{2}\right)\right) \hat{(}\left(x_{p}\right)\right| \\
& -\left|\left(D\left(\sum_{i=1}^{p-1}\left(\prod_{j=1}^{i} f_{j}^{2}\right) F_{i}\right)\right) \hat{(}\left(x_{p}\right)\right| .
\end{aligned}
$$

Then by 2) we have

$$
\begin{aligned}
\left|(D(G)) \hat{(}\left(x_{p}\right)\right| & \geqq p-\left\|F_{p}\right\|_{A}\left\|D\left(\prod_{j=1}^{p} f_{j}^{2}\right)\right\|_{A} \\
& \geqq p-1 .
\end{aligned}
$$

We conclude that $\left|(D(G))^{\wedge}\left(x_{p}\right)\right| \geqq p-1$, which is a contradiction since $(D(G))^{\wedge}$ is a bounded function on $M_{A}$.

\section{Main results.}

In this section we consider the problem on the image of a ring derivation on a commutative Banach algebra. In the case of a radical algebra the image is of course contained in the radical, so we consider the case of the algebra with a non-zero complex homomorphism. Suppose that $A$ is a semi-simple commutative Banach algebra with the carrier space $M_{A}$ and $x_{1}, \cdots, x_{n}$ are isolated points in $M_{A}$. Then there are idempotents $e_{1}, \cdots, e_{n}$ in $A$ such that $\hat{e}_{i}(x)=1$ for $x=x_{i}$ and for otherwise $\hat{e}_{i}(x)=0$ for each $i$. (This is a direct consequence of the Silov idempotent theorem.) Suppose also that $D_{1}, \ldots, D_{n}$ are ring derivations on $C$. Then an operator $D$ defined by $D(f)=\sum_{i=1}^{n} D_{i}\left(f\left(x_{i}\right)\right) e_{i}$ is a ring derivation on $A$. We consider the converse of the fact. As a consequence of the following theorem the converse is also true for semi-simple commutative Banach algebras, that is, a ring derivation on a semi-simple commutative Banach algebra has such a representation as above.

TheOREM. Let $A$ be a commutative Banach algebra with the carrier space $M_{A}$. Let $D$ be a ring derivation on $A$. We assume the following:

$$
D(\operatorname{rad}(A)) \subset \operatorname{rad}(A),
$$

where $\operatorname{rad}(A)$ is the (Jacobson) radical of $A$. Then there are at most finite number of isolated points in $M_{A}$, say $y_{1}, \cdots, y_{n}$, and the same number of ring derivations $D_{1}, \cdots, D_{n}$ on the complex number field which satisfy: 


$$
D(f) \in \sum_{i=1}^{n} D_{i}\left(\hat{f}\left(y_{i}\right)\right) e_{i}+\operatorname{rad}(A),
$$

where $e_{i}$ is an idempotent such that $\hat{e}_{i}(x)=1$ for $x=y_{i}$ and $\hat{e}_{i}(x)=0$ for $x \neq y_{i}$ for every $i$.

Proof. Let $\left\{y_{1}, \cdots, y_{n}\right\}$ be a set of points $x$ in $M_{A}$ at which the functional $\phi_{x}$ is discontinuous, then the set is finite by Lemma 5 . First we show that $(D(f))^{\wedge}$ vanishes off $\left\{y_{1}, \cdots, y_{n}\right\}$ for every $f$ in $A$ and each $y_{i}$ is an isolated point in $M_{A}$. Put $\hat{A}=\{\hat{f}: f \in A\}$. Then $\hat{A}$ is a semi-simple Banach algebra, with respect to the quotient norm induced by $A / \operatorname{rad}(A)$, of which the carrier space is $M_{A}$. Put $K=$ the closure of $M_{A}-\left\{y_{1}, \cdots, y_{n}\right\}$ in $M_{A}$. Then $\hat{A} \mid K$ is a Banach algebra with respect to the quotient norm. We define an operator $\tilde{D}$ on $\hat{A} \mid K$ by $\tilde{D}(\varphi)=(D(f))^{\wedge} \mid K$, where $\varphi=\hat{f} \mid K$ for some $f$ in $A$. Then $\tilde{D}$ is well defined and is a ring derivation on $\hat{A} \mid K$. We will show that $\tilde{D}$ is well defined. Suppose that $\left\{y_{i(1)}, \cdots, y_{i(l)}\right\}=M_{A}-K$. Then $\left\{y_{i(1)}, \cdots, y_{i(l)}\right\}$ is a subset of $\left\{y_{1}, \cdots, y_{n}\right\}$ and each $y_{i(j)}$ is an isolated point in $M_{A}$ and so for every $j$ there is an idempotent $e_{i(j)}$ in $A$ such that $\hat{e}_{i(j)}(x)=0$ for $x \neq y_{i(j)}$ and $\hat{e}_{i(j)}\left(y_{i(j)}\right)=1$. Suppose that $\hat{f}|K=\hat{g}| K$. Then we see that

$$
f-g=\sum_{j=1}^{l}\left(\hat{f}\left(y_{i(j)}\right)-\hat{g}\left(y_{i(j)}\right)\right) e_{i(j)}+r,
$$

where $r$ is in $\operatorname{rad}(A)$. So we have

$$
\begin{aligned}
D(f-g) & =\sum_{j=1}^{l} D\left(\left(\hat{f}\left(y_{i(j)}\right)-\hat{g}\left(y_{i(j)}\right)\right) e_{i(j)}\right)+D(r) \\
& =\sum_{j=1}^{l} D\left(\left(\hat{f}\left(y_{i(j)}\right)-\hat{g}\left(y_{i(j)}\right)\right) e_{i(j)}^{2}\right)+D(r) \\
& =\sum_{j=1}^{l} D\left(\left(\hat{f}\left(y_{i(j)}\right)-\hat{g}\left(y_{i(j)}\right)\right) e_{i(j)}\right) e_{i(j)}+D(r),
\end{aligned}
$$

since $e_{i(j)}=e_{i(j)}^{2}$ and $D\left(e_{i(j)}\right)=0$. (If $e$ is an idempotent in $A$, then $D(e)=0$ since $2 e D(e)=D\left(e^{2}\right)=D(e)$ and $2 e D(e)=2 e^{2} D(e)=e D\left(e^{2}\right)=e D(e)$.) It follows by $\left.*\right)$ that

$$
(D(f-g))^{\wedge}=\sum_{j=1}^{l}\left(D\left(\left(\hat{f}\left(y_{i(j)}\right)-\hat{g}\left(y_{i(j)}\right)\right) e_{i(j)}\right)\right)^{\wedge} \hat{e}_{i(j)}
$$

and so we have $(D(f))^{\wedge}\left|K=(D(g))^{\wedge}\right| K$, that is, $\tilde{D}$ is well defined. The fact that $\tilde{D}$ is a ring derivation is easy to prove. If we can prove that $\tilde{D}$ is linear, then since $\hat{A} \mid K$ is semi-simple we have $\tilde{D}=0$ by the fact that there are no nonzero continuous linear derivations on semi-simple commutative Banach algebras (cf. [5, Theorem 1], [2, Theorem 2], [6]). It follows that $(D(f))^{\wedge} \mid K=0$ for every $f$ in $A$. We then see that $\phi_{x}=0$, that is, $\phi_{x}$ is continuous for every $x$ in $K$. We also conclude that

$$
\left\{y_{1}, \cdots, y_{n}\right\}=\left\{y_{i(1)}, \cdots, y_{i(l)}\right\} \text {. }
$$

Therefore each $y_{i}$ is an isolated point in $M_{A}$ and $(D(f))^{\wedge}$ vanishes off $\left\{y_{1}, \cdots, y_{n}\right\}$ for 
every $f$ in $A$.

We will prove that $\tilde{D}$ is linear. Let $x$ be a point in $M_{A}-\left\{y_{1}, \cdots, y_{n}\right\}$. We show that $\phi_{x}$ is linear, that is, $\phi_{x}(\alpha f)=\alpha \phi_{x}(f)$ for every complex number $\alpha$ and $f$ in $A$. Choose a sequence $\left\{\alpha_{n}\right\}$ of rational complex numbers such that $\alpha_{n} \rightarrow \alpha$. Then $\phi_{x}\left(\left(\alpha-\alpha_{n}\right) f\right) \rightarrow 0$ since $\phi_{x}$ is continuous. On the other hand

$$
\begin{aligned}
\phi_{x}\left(\left(\alpha-\alpha_{n}\right) f\right) & =\left(D\left(\left(\alpha-\alpha_{n}\right) f\right)\right)^{\wedge}(x) \\
& =(D(\alpha f))^{\wedge}(x)-\left(D\left(\alpha_{n} f\right)\right)^{\wedge}(x) \\
& =(D(\alpha f))^{\wedge}(x)-\alpha_{n}(D(f))^{\wedge}(x) \\
& =\phi_{x}(\alpha f)-\alpha_{n} \phi_{x}(f)
\end{aligned}
$$

by Lemma 1. Since $\alpha_{n} \phi_{x}(f) \rightarrow \alpha \phi_{x}(f)$ we conclude that $\phi_{x}(\alpha f)=\alpha \phi_{x}(f)$. Thus we have $(D(\alpha f))^{\wedge}(x)=\alpha(D(f))^{\wedge}(x)$ on $M_{A}-\left\{y_{1}, \cdots, y_{n}\right\}$, and so on $K$. It follows that $\tilde{D}$ is a linear derivation.

For $1 \leqq i \leqq n$ define the ring derivation $D_{i}$ on the complex number field by

$$
D_{i}(\alpha)=\left(D\left(\alpha e_{i}\right)\right)^{\wedge}\left(y_{i}\right),
$$

where $e_{i}$ is an idempotent in $A$ such that $\hat{e}_{i}\left(y_{i}\right)=1$ and $\hat{e}_{i}(x)=0$ for $x \neq y_{i}$. Note that $D_{i}$ is well defined since $\left(D\left(\alpha e_{i}\right)\right)^{\wedge}=\left(D\left(\alpha e_{i}^{\prime}\right)\right)^{\wedge}$ holds for idempotents $e_{i}$ and $e_{i}^{\prime}$ in $A$ with $\hat{e}_{i}=\hat{e}_{i}^{\prime}$ by the condition $*$ ). Since $D(e)=0$ for an idempotent $e$ we see that $D\left(f-\sum_{i=1}^{n} f e_{i}\right)$ is in $\operatorname{rad}(A)$ for every $f$ in $A$. For $\left(D\left(f-\sum_{i=1}^{n} f e_{i}\right)\right)^{\wedge}$ vanishes off $\left\{y_{1}, \cdots, y_{n}\right\}$ and

$$
\begin{aligned}
\left(D\left(f-\sum_{i=1}^{n} f e_{i}\right)\right)^{\wedge}\left(y_{j}\right) & =(D(f))^{\wedge}\left(y_{j}\right)-\sum_{i=1}^{n}\left(D\left(f e_{i}\right)\right)^{\wedge}\left(y_{j}\right) \\
& =(D(f))^{\wedge}\left(y_{j}\right)-\sum_{i=1}^{n}(D(f))^{\wedge}\left(y_{j}\right) \hat{e}_{i}\left(y_{j}\right) \\
& =0
\end{aligned}
$$

for $1 \leqq j \leqq n$, we have that $\left(D\left(f-\sum_{i=1}^{n} f e_{i}\right)\right)^{\wedge}$ vanishes on $M_{A}$. We have $D\left(f e_{i}-\hat{f}\left(y_{i}\right) e_{i}\right)$ is in $\operatorname{rad}(A)$ since $f e_{i}-\hat{f}\left(y_{i}\right) e_{i}$ is in $\operatorname{rad}(A)$ and the condition $\left.*\right)$ holds. We also see that

$$
D\left(\hat{f}\left(y_{i}\right) e_{i}\right)-\left(D\left(\hat{f}\left(y_{i}\right) e_{i}\right)\right)^{\wedge}\left(y_{i}\right) e_{i}
$$

is in the radical of $A$. It follows that

$$
\begin{aligned}
D(f)= & D\left(f-\sum_{i=1}^{n} f e_{i}\right)+D\left(\sum_{i=1}^{n}\left(f e_{i}-\hat{f}\left(y_{i}\right) e_{i}\right)\right) \\
& +\sum_{i=1}^{n}\left\{D\left(\hat{f}\left(y_{i}\right) e_{i}\right)-\left(D\left(\hat{f}\left(y_{i}\right) e_{i}\right)\right)^{\wedge}\left(y_{i}\right) e_{i}\right\}+\sum_{i=1}^{n}\left(D\left(\hat{f}\left(y_{i}\right) e_{i}\right)\right)^{\wedge}\left(y_{i}\right) e_{i}
\end{aligned}
$$

is in

$$
\sum_{i=1}^{n}\left(D\left(\hat{f}\left(y_{i}\right) e_{i}\right)\right)^{\wedge}\left(y_{i}\right) e_{i}+\operatorname{rad}(A)=\sum_{i=1}^{n} D_{i}\left(\hat{f}\left(y_{i}\right)\right) e_{i}+\operatorname{rad}(A)
$$


COROllary 1. Let $A$ be a semi-simple commutative Banach algebra. Let $D$ be a ring derivation on $A$. Then there exist at most finite number of isolated points $y_{1}, \cdots, y_{n}$ in the carrier space $M_{A}$ and the same number of ring derivations $D_{1}, \cdots, D_{n}$ on the complex number field which satisfy that $D(f)=\sum_{i=1}^{n} D_{i}\left(\hat{f}\left(y_{i}\right)\right) e_{i}$ for every $f$ in $A$, where $e_{i}$ is the idempotent in $A$ such that $\hat{e}_{i}\left(y_{i}\right)=1$ and $\hat{e}_{t}(x)=0$ for $x \neq y_{i}$.

Since a function algebra is a semi-simple commutative Banach algebra we see that every ring derivation on a function algebra is represented as in the same way as in Corollary 1 (cf. [3], [4]).

COROLlARY 2. Let A be a semi-simple commutative Banach algebra with the carrier space without isolated points. Then only the zero operator is the ring derivation on $A$.

\title{
References
}

[1] P. Cartífr, Derivations dans les corps, Géometrique Algébrique, Séminaire E. N. S., 8 (1955/56).

[2] B. E. Johnson, Continuity of derivations on commutative algebras, Amer. J. Math., 91 (1969), 1-10.

[ 3 ] O. HATORI and J. WADA, A characterization of ring derivations on function algebras, Bull. Tokyo Med. Coll., 17 (1991), 19-22.

[4] N. R. NANDAKumar, Ring derivations on function algebras, Canad. Math. Bull., 33 (1990), 69-72.

[ 5] I. M. Singer and J. WERMER, Derivations on commutative algebras, Math. Ann., 129 (1955), $260-264$.

[6] M. P. Thomas, Image of a derivation is contained in the radical, Ann. of Math., 128 (1988), $435-460$.

\author{
Present Address: \\ OSAMU HATORI \\ Department of Mathematics, Tokyo Medical College \\ 6-1-1 Shinjuku, Shinjuku-Ku, TOKyo 160, JAPAN \\ e-mail : berobero@jpnwas00 \\ JUNZO WADA \\ Department of Mathematics, School of Education, Waseda University \\ Nishi-Waseda, ShinJUkU-KU, TOKYo 169-50, JAPAN
}

